\title{
Spectrum of retinal abnormalities in renal transplant patients using chronic low-dose steroids
}

\author{
Elon H. C. van Dijk ${ }^{1}$ - Darius Soonawala ${ }^{2,3}$ - Vera Rooth ${ }^{1}$ - Carel B. Hoyng ${ }^{4}$. \\ Onno C. Meijer ${ }^{5,6}$ • Aiko P. J. de Vries ${ }^{2}$ Camiel J. F. Boon ${ }^{1,7}$
}

Received: 31 July 2017 /Revised: 20 September 2017 / Accepted: 5 October 2017 /Published online: 23 October 2017

(C) The Author(s) 2017. This article is an open access publication

\begin{abstract}
Purpose To assess the ophthalmological characteristics of asymptomatic patients with a renal transplant on chronic low-dose steroids for at least the last 2 years prior to examination.

Methods Cross-sectional study. All patients underwent an extensive ophthalmological examination.

Results Of the 37 included patients 25 male, 12 female; $59 \pm 11$ years (range, 38-77 years)] ophthalmological phenotyping revealed abnormalities in 22 patients (59\%). Findings characteristic for (subclinical) central serous chorioretinopathy were detected in ten patients (27\%), including two patients with serous subretinal fluid in the macula. An
\end{abstract}

Camiel J. F. Boon

c.j.f.boon@lumc.nl

1 Department of Ophthalmology, Leiden University Medical Center, Albinusdreef 2, 2333 ZA Leiden, the Netherlands

2 Department of Medicine, Division of Nephrology, and Leiden Transplant Center, Leiden University Medical Center, Leiden, the Netherlands

3 Department of Medicine, Division of Nephrology, Haga Hospital, the Hague, the Netherlands

4 Department of Ophthalmology, Donders Institute of Brain, Cognition, and Behaviour, Radboud University Medical Center, Nijmegen, the Netherlands

5 Department of Medicine, Division of Endocrinology and Metabolism, Leiden University Medical Center, Leiden, the Netherlands

6 Einthoven Laboratory for Experimental Vascular Medicine, Leiden University Medical Center, Leiden, the Netherlands

7 Department of Ophthalmology, Academic Medical Center, University of Amsterdam, P.O. Box 9600, 2300 RC Leiden, The Netherlands epiretinal membrane of the macula was present in six patients (16\%). Mean subfoveal choroidal thickness was significantly increased in patients with ophthalmological abnormalities, in comparison with patients without abnormalities.

Conclusions Retinal abnormalities are common in the majority of renal transplant patients using chronic low-dose steroids. These retinal changes may be associated with the renal disease and/or the effect of chronic steroid use on the choroid and retina.

Keywords Cross-sectional study · Renal transplant . Ophthalmological examination $\cdot$ Retinal phenotyping · Steroid use

\section{Introduction}

For end-stage solid organ disease, transplantation is a therapeutic option in a noteworthy number of patients. Abnormal ophthalmological findings after several types of transplantation occur in more than $50 \%$ of patients [1-3], and are caused by the combination of pre-existing disease and immunosuppressive regimen [4]. Fortunately, vision-threatening conditions are seldom observed after long-term follow-up [5]. Up to $45 \%$ of the transplant patients develop typical steroidinduced bilateral posterior subcapsular cataract within the first year after transplantation [1]. Bilateral, open-angle glaucoma is detected in up to $7 \%$ of patients within the first year after transplantation, which may be caused by the use of systemic steroids [1]. Little is known about transplantation complications in the posterior segment of the eye. Documented retinal complications are of infectious, hemorrhagic, or microvascular origin and have been described after bone marrow transplantation preceded by total body irradiation [4]. Moreover, optic disc edema and pigmentary changes have been found to 
occur after heart transplantation [6]. The kidney is the most commonly transplanted organ worldwide. Little is known about retinal abnormalities in renal transplant patients. Some renal diseases that often require renal transplantation, for example complement-associated diseases like dense deposit disease, are known to be associated with retinal abnormalities such as drusen and macular degeneration [7]. Use of steroids as is common after transplantation, is known to be a major risk factor associated with central serous chorioretinopathy (CSC). This is a form of macular degeneration caused by an accumulation of serous subretinal fluid (SRF) due to choroidal and retinal pigment epithelium (RPE) abnormalities [8-11]. Previous steroid intake has been described in up to $52 \%$ of CSC patients, and odds ratios of up to 37 for CSC have been found for steroid using subjects $[8,12,13]$.

In the current study, we performed an extensive crosssectional ophthalmological phenotyping in renal transplant patients on low-dose steroids for at least 2 years prior to examination.

\section{Methods}

\section{Patient selection}

In this cross-sectional study, 37 sequential outpatient renal transplant patients who chronically used oral low-dose steroids for at least 2 years prior to examination and who did not have visual complaints were invited to take part in this study conducted at the Department of Ophthalmology of the Leiden University Medical Center, for ophthalmological analysis. Patients who were previously diagnosed with either retinal abnormalities or systemic disorders that may cause persistent retinal damage (for example uncontrolled diabetes mellitus or uncontrolled hypertension) were excluded. Written informed consent was signed by all subjects. The study adhered to the tenets of the Declaration of Helsinki, and was approved by the institutional review board and the ethics committee (NL50816.058.14). Clinical information was collected from the patients' charts. Patients were included in this study from February 2016 to October 2016.

\section{Ophthalmological imaging}

Patients underwent an extensive ophthalmic assessment, including Early Treatment of Diabetic Retinopathy Study (ETDRS) best-corrected visual acuity (BCVA) measurement, and indirect ophthalmoscopy. After administration of $1 \%$ tropicamide and 5\% phenylephrine drops, digital color fundus photography of the central and peripheral retina (Topcon Corp., Tokyo, Japan) was performed, and optical coherence tomography (OCT), enhanced depth imaging (EDI-)OCT, fundus autofluorescence, and oral fluorescein angiography (FA) images were made with the spectral-domain OCT
(Spectralis HRA + OCT; Heidelberg Engineering, Dublin, CA, USA). Subfoveal choroidal thickness (SFCT; distance from the outer part of the hyperreflective RPE layer to the hyperreflective line of the inner surface of the sclera) was measured on EDI-OCT. For the oral FA, patients had to ingest $10 \mathrm{ml}$ of $20 \%$ fluorescein after a fasting period of at least $3 \mathrm{~h}$. Oral FA images were obtained at 10, 15, 20, 25, and $30 \mathrm{~min}$ after the administration of fluorescein. Peripheral images were acquired between 25 and $30 \mathrm{~min}$ after ingestion. When the evaluation of ophthalmological imaging by an experienced retina specialist (CJFB) led to findings requiring treatment and/or follow-up, these examinations and/or visits were scheduled at the outpatient clinic of the Department of Ophthalmology of Leiden University Medical Center.

\section{Statistical analysis}

For statistical analyses, descriptive statistics were used in SPSS Statistics (version 23; IBM Corp., Armonk, NY, USA). Independent $t$-tests were used to compare the patient group in whom ophthalmological abnormalities could be detected to the remaining patients for: need for and total duration of dialysis before transplantation, reason of (re)transplantation, time from the first renal transplantation (and start of steroid use) until phenotyping, number of rejection therapies, type of immunosuppressive drugs at the moment of phenotyping, age of the patient at the moment of transplantation and at phenotyping, and donor age at the moment of transplantation. Moreover, independent $t$-tests were used to compare the mean SFCT for patients with and without ophthalmological abnormalities. The level of statistical significance was set at $p<0.05$.

\section{Results}

\section{Patient characteristics}

The mean age at phenotyping of the 37 included patients ( 25 male, 12 female) was $59 \pm 11$ years (range, 38-77 years). The mean age at which the first renal transplantation had been performed was $50 \pm 12$ years (range, 22-71 years). Retransplantation had been performed in four patients $(11 \%)$. Eleven patients received a transplantation prior to dialysis. The total duration of dialysis was $3.2 \pm 2.1$ years (range, 0.3-7.5 years) in the 24 other patients, for whom this information was available.

Among other immunosuppressant medication, which was used by all patients, all patients started using steroids after their first transplantation. The estimated minimal cumulative prednisolone dose was $4000 \mathrm{mg}$ and the mean cumulative prednisolone dose was $20,000 \mathrm{mg}$. At the moment of ophthalmological phenotyping, 25 patients used $5 \mathrm{mg}$ and 12 used $7.5 \mathrm{mg}$ prednisolone once daily. Eleven patients reported the 
use of other steroid-containing medication during the last year before ophthalmological phenotyping. The medical history of the participants was notable for arterial hypertension in all patients (100\%) and diabetes mellitus in 12 patients (32\%). Besides the diagnosis of a depression in three patients (8\%), no other diseases known to be possibly associated with CSC were present [14]. Four patients used a mineralocorticoid receptor antagonist to treat hypertension, a drug which has also been described as a therapeutic option for CSC [15]. Clinical characteristics of the patients are summarized in Table 1.

\section{Ophthalmic characteristics}

At the visit to the Department of Ophthalmology, ETDRS BCVA was $84 \pm 11$ letters (range, 25-96 letters) in the included eyes. Two patients had a history of unilateral amblyopia, and one patient of recurrent unilateral herpes keratitis. In 60 eyes (79\%) ETDRS BCVA was $\geq 80$ letters. During slit-lamp examination, unilateral mature cataract was detected in one patient. BCVA ETDRS in this patient improved from 25 to 85 ETDRS letters, after phacoemulsification. Besides the presence of pre-existing primary open-angle glaucoma in one patient, no other abnormalities of the anterior segment were found. OCT showed the presence of macular serous SRF in three eyes of two patients (5\%). On FA, hyperfluorescent changes that could fit well within the spectrum of (subclinical) CSC were detected in these eyes, without the presence of a 'hot spot' of leakage (Fig. 1a-e). Fundus autofluorescence revealed variable mild hypo- and hyperfluorescence of the lesions. On OCT imaging, a variable degree of outer photoreceptor/RPE changes compatible with subclinical CSC was found in eight of the remaining patients (22\%), which was supported by corresponding hyperfluorescent changes on FA (Fig. 1f-j). On OCT imaging, two patients had a bilateral epiretinal membrane (ERM), and four had a unilateral ERM, resulting in a total of six patients $(16 \%)$ in whom an ERM in the macula was detected (Fig. 1w$\mathrm{x})$. One of the patients with a unilateral ERM also had a macular pseudohole. Cuticular drusen, which are associated with membranoproliferative glomerulonephritis type II (dense deposit disease), were seen in one patient with this disease (Fig. $1 \mathrm{k}-\mathrm{o}$ ), and multiple, small yellow-white lesions in the midperiphery of the fundi, which are associated with Alport's disease, were seen in one patient with this diagnosis (Fig. 1uv) $[16,17]$. Bilateral intermediate age-related macular degeneration with soft drusen and secondary vitelliform-like lesions was found in one patient (Fig. 1p-t), whereas bilateral RPE detachments in combination with drusenoid lesions were detected in another patient. In single patients, a unilateral RPE detachment, an old unilateral retinal venous occlusion, and
Table 1 Clinical characteristics of the 37 phenotyped patients who chronically used low-dose steroids for at least the last 2 years prior to examination

\begin{tabular}{ll}
\hline Clinical characteristics & \\
\hline Number of patients & 37 \\
Number of males & $25(68 \%)$ \\
Number of females & $12(32 \%)$ \\
Mean age \pm SD (range) at phenotyping, in years & $59 \pm 11(38-77)$ \\
Mean age \pm SD (range) at renal transplantation, in years & $50 \pm 12(22-71)$ \\
& \\
Reason for first transplantation & \\
Autosomal polycystic kidney disease & $8(22 \%)$ \\
IgA nephropathy & $7(19 \%)$ \\
Unknown origin of disease & $6(16 \%)$ \\
Acute tubular necrosis & $2(5 \%)$ \\
Membranoproliferative glomerulonephritis & $2(5 \%)$ \\
Malignancy & $2(5 \%)$ \\
Reflux nephropathy & $2(5 \%)$ \\
Alport syndrome & $1(3 \%)$ \\
C3 glomerulopathy & $1(3 \%)$ \\
Chronic urinary tract infections & $1(3 \%)$ \\
Hemolytic-uremic syndrome + hypertension & $1(3 \%)$ \\
Medullary cystic kidney disease & $1(3 \%)$ \\
Oxalate nephropathy & $1(3 \%)$ \\
Pauci-immune crescentic glomerulonephritis & $1(3 \%)$ \\
Tubulointerstitial nephritis & $1(3 \%)$ \\
Retransplantation & $4(11 \%)$ \\
\hline
\end{tabular}



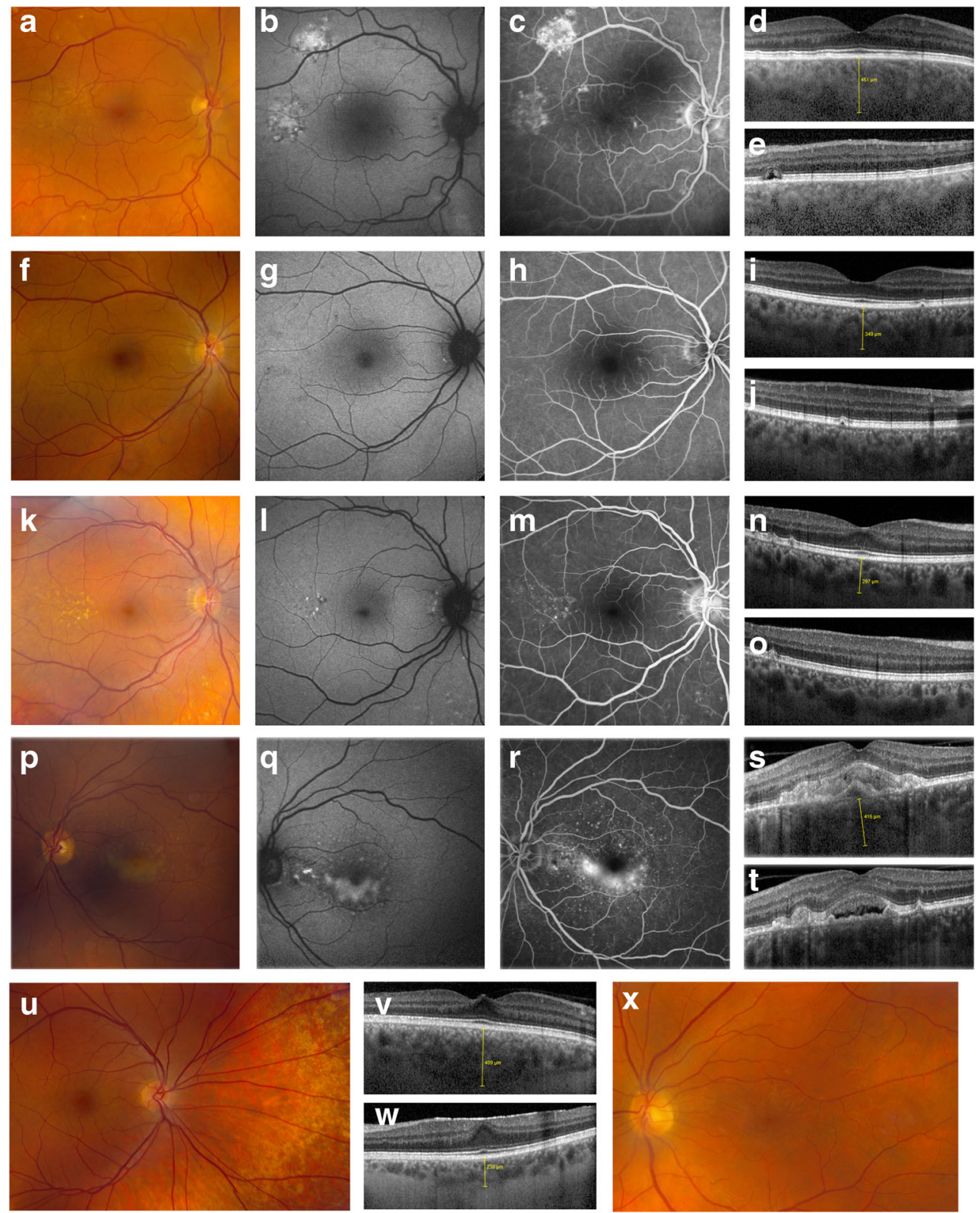

unilateral RPE atrophy were detected. In patients with (subclinical) CSC, mean SFCT on EDI-OCT was $286 \pm 82 \mu \mathrm{m}$ (range, 111-451 $\mu \mathrm{m}$ ). In all eyes with retinal abnormalities, mean SFCT on EDI-OCT was $301 \pm 88 \mu \mathrm{m}$ (range, 111-489 $\mu \mathrm{m}$ ), and mean SFCT in all eyes of patients with either unilateral or bilateral abnormalities was $292 \pm 84 \mu \mathrm{m}$ (range, 111-489 $\mu \mathrm{m}$ ). These CTs differed from the eyes of patients in whom no abnormalities were detected (232 $\pm 108 \mu \mathrm{m}$ (range, 98-571 $\mu \mathrm{m}$ ); $p=0.071, p=0.01$, and $p=0.006$ respectively).

None of the clinical characteristics of the included patients could be linked with either the presence or absence of ocular abnormalities. Treatment was not needed for the retinal abnormalities that were detected in this study. For the patient with macular pseudohole and for the patient with maculopathy with secondary vitelliformlike lesions, additional follow-up visits were scheduled. The retinal findings of the patients are summarized in Table 2.

\section{Discussion}

Retinal abnormalities were found in 22 of 37 renal transplant patients (59\%), who were using chronic low-dose steroids for at least 2 years prior to examination and who were not previously known to have chorioretinal diseases. Findings characteristic for (subclinical) CSC were found in ten patients (27\%), and two of these patients showed active SRF leakage in the macula. Moreover, in five other patients and in a total of six 
4 Fig. 1 Spectrum of retinal abnormalities in renal transplant patients who chronically used low-dose steroids for at least the last 2 years prior to examination. a-e Multimodal imaging of a 58-year-old male patient who received a renal transplant 21 years before phenotyping, because of membranoproliferative glomerulonephritis. Fundus photography of the right eye (a) showed mild foveal and extrafoveal retinal pigment epithelium (RPE) abnormalities, and on fundus autofluorescence (FAF; b) irregular changes were present. Fluorescein angiography of the right eye (FA; c), which was performed after oral administration of fluorescein, showed multifocal hyperfluorescent areas, but no clear 'hot spot' of leakage. On the foveal optical coherence tomography (OCT) scan of the right eye (d) RPE and outer photoreceptor changes suggestive of central serous chorioretinopathy, and a subfoveal choroidal thickness (SFCT) of $451 \mu \mathrm{m}$ were found. No subretinal fluid (SRF) was present in the right eye. In the left eye, milder abnormalities were detected on multimodal imaging, but extrafoveal OCT scanning (e) showed SRF nasosuperiorly and RPE changes. $\mathbf{f}-\mathbf{j}$ Multimodal imaging of a 43 -year-old male patient who was transplanted 11 years ago, because of reflux nephropathy. On fundus photography of the right eye (f) subtle extrafoveal RPE abnormalities were observed, with corresponding changes on FAF (g). Mild hyperfluorescent changes were found on oral FA (h) of this eye, partly due to staining and partly due to a window defect. The foveal (i) and extrafoveal OCT scans (j) revealed mild outer retinal changes and the SFCT was $349 \mu \mathrm{m}$. Moreover, RPE detachments, which could also correspond to age-related (subclinical) small hard drusen, were found. Similar findings were observed in the left eye of this patient. $\mathbf{k}-\mathbf{o}$ Multimodal imaging of a 44-year-old female patient who underwent transplantation surgery 22 years before retinal phenotyping, and had received a transplant because of membranoproliferative glomerulonephritis. Cuticular drusen, known to be associated with membranoproliferative glomerulonephritis type II (dense deposit disease), were detected on fundus photography (k), FAF (I), oral FA $(\mathbf{m})$, and both foveal (n) and extrafoveal OCT images (o) of the right eye. SFCT was $297 \mu \mathrm{m}$ at this visit. In the left eye, identical findings were detected. p-t Multimodal imaging of a 63 -year-old male patient who received a renal transplant 6 years before ophthalmological phenotyping, because of IgA nephropathy, showed findings characteristic for bilateral nonexudative age-related macular degeneration and secondary vitelliform-like lesions. On fundus photography of the left eye (p), yellow-white lesions and confluent drusen were seen in the macula, together with cuticular drusen and RPE alterations peripherally. On FAF (q) hyperfluorescent changes with an irregular border were seen in the macular area. Oral FA (r) revealed cuticular drusen and irregular hyperfluorescent changes foveally and perifoveally, which could be characteristic for the vitelliform-like lesions. Foveal (s) and extrafoveal OCT images (t) showed drusen, and a hyperreflective subretinal accumulation, that was most pronounced foveally. Comparable lesions were found in the other eye of this patient. $\mathbf{u}-\mathbf{v}$ In a 37-year-old male patient, who had received a renal transplant for Alport's disease 7 years before retinal phenotyping, fundus photography of the right eye (u) showed multiple, small yellow-white lesions in the midperiphery of the fundi, known to be associated with this disease. Foveal OCT scanning (v) showed some traction of unknown origin, and a SFCT of $489 \mu \mathrm{m}$. Comparable lesions were found in the left eye. $\mathbf{w}-\mathbf{x}$ In a 62-year-old female patient, who was previously diagnosed with autosomal dominant polycystic kidney disease and was transplanted 19 years ago, a unilateral foveal epiretinal membrane was detected on OCT (w). SFCT was $238 \mu \mathrm{m}$, at that visit. The epiretinal membrane was also observed on fundus photography (x)

patients $(16 \%)$ an ERM was found. These findings indicate that renal transplant patients could benefit from ophthalmological examination. The frequent occurrence of CSC-like abnormalities may have been caused by steroid use, which has been described to be its most important risk factor [12, 18, 19]. In light of the underlying disease and use of comedication, and the cross-sectional/observational nature of this study, it remains uncertain whether a causal relation exists between the use of steroids and the high prevalence of retinal abnormalities in renal transplant patients. If there is a causal relation, this might be either due to a (previous) short-term high dosage or due to the cumulative effect of a long-term low dosage.

Extensive ophthalmological phenotyping in renal transplant patients using steroids has not been conducted so far. The low prevalence of CSC with active SRF leakage in our cohort (5\%) is surprising, given the fact that all patients had used high cumulative doses of steroids. This suggests that the susceptibility for developing CSC depends on more risk factors than solely the use of steroids. Still, $22 \%$ of patients had retinal abnormalities that may compatible with subclinical CSC without SRF leakage. In a previous study, in which the authors did not exclude patients with pre-existing ophthalmological diseases, and in which no ophthalmological imaging was performed and in which exact definitions of disease were lacking, retinal pigment epitheliopathy (14\%), CSC (3\%), retinal vein occlusion (3\%), and macular edema (3\%) were described [11]. The number of patients with retinal pigment epitheliopathy/CSC in our study seems to be in line with these previously published findings.

For this study, only patients who used steroids for more than 2 years and who did not have a history of either chorioretinal diseases or complaints that could be related to these diagnoses were included. Since a selection based on underlying renal pathology was not performed, patients with various renal diseases were included. In contrast with another study, we did not find a correlation between the outcome of phenotyping and the duration of dialysis before transplantation and time since transplantation [11]. Other clinical characteristics of the included patients could also not be linked to the occurrence of ophthalmological abnormalities. However, compared to patients in whom no ophthalmological abnormalities were found, a significantly increased mean SFCT was observed in patients in whom ophthalmological abnormalities were seen. In CSC patients, the choroid is usually thicker compared to the healthy population, strengthening the relevance of this finding [20-22]. Both the increased CT and percentage of patients with (subclinical) CSC in this study could thus have been caused by the effect of steroids on the choroid, in cases of administration for a prolonged period and only in a specific patient group with predisposition to develop these abnormalities [23, 24]. However, CT between patients with (subclinical) CSC and patients without ophthalmological abnormalities did not differ significantly, which may be related to the small sample size. Since younger age, shorter axial length, male gender, deeper anterior chamber, thicker lens, flatter cornea, and better BCVA have all been associated with 
Table 2 Reason for transplantation of the 22 phenotyped patients with retinal abnormalities who chronically used low-dose steroids for at least the last 2 years prior to examination

\begin{tabular}{|c|c|}
\hline Retinal abnormality & Reason for transplantation (number of patients) \\
\hline $\begin{array}{l}\text { Bilateral findings characteristic for CSC*, } \\
\text { bilateral SRF }\end{array}$ & Membranoproliferative glomerulonephritis (1) \\
\hline $\begin{array}{l}\text { Bilateral findings characteristic for CSC*, } \\
\text { unilateral SRF }\end{array}$ & Unknown origin of disease (1) \\
\hline Bilateral findings characteristic for CSC*, no SRF & $\begin{array}{l}\text { Acute tubular necrosis after surgery (1), ADPKD (1), } \\
\text { reflux nephropathy (1), tubulointerstitial nephritis (1) }\end{array}$ \\
\hline Unilateral findings characteristic for $\mathrm{CSC}^{*}$, no SRF & $\begin{array}{l}\text { Chronic urinary tract infections (1), hemolytic-uremic } \\
\text { syndrome AND hypertension (1), IgA nephropathy } \\
\text { (1) }\end{array}$ \\
\hline $\begin{array}{l}\text { Unilateral findings characteristic for } \mathrm{CSC}^{*} \text {, no SRF; } \\
\text { AND a bilateral epiretinal membrane }\end{array}$ & ADPKD (1) \\
\hline Bilateral epiretinal membrane & Unknown origin of disease (1) \\
\hline Unilateral epiretinal membrane & $\begin{array}{l}\text { ADPKD (2), pauci-immune crescentic } \\
\text { glomerulonephritis (1) }\end{array}$ \\
\hline $\begin{array}{l}\text { Unilateral epiretinal membrane AND a unilateral } \\
\text { macular pseudohole AND bilateral choroidal folds }\end{array}$ & Nephrosclerosis AND neoplasm (1) \\
\hline $\begin{array}{l}\text { Bilateral cuticular drusen, known for } \\
\text { membranoproliferative glomerulonephritis }\end{array}$ & Membranoproliferative glomerulonephritis (1) \\
\hline $\begin{array}{l}\text { Bilateral dry age-related macular degeneration } \\
\text { AND secondary vitelliform lesions AND } \\
\text { cuticular drusen }\end{array}$ & IgA nephropathy (1) \\
\hline $\begin{array}{l}\text { Bilateral peripheral yellow-white lesions, } \\
\text { known for Alport syndrome }\end{array}$ & Alport syndrome (1) \\
\hline $\begin{array}{l}\text { Bilateral retinal pigment epithelium detachments } \\
\text { AND drusenoid lesions }\end{array}$ & Oxalate nephropathy (1) \\
\hline Unilateral old venous occlusion & Unknown origin of disease (1) \\
\hline Unilateral retinal pigment epithelium atrophy & Unknown origin of disease (1) \\
\hline Unilateral retinal pigment epithelium detachment & ADPKD (1) \\
\hline No abnormalities & $\begin{array}{l}\text { Acute tubular necrosis after surgery (1), ADPKD (3), } \\
\text { C3 glomerulopathy (1), IgA nephropathy (5), } \\
\text { medullary cystic kidney disease (1), nephrosclerosis } \\
\text { AND neoplasm (1), reflux nephropathy (1), } \\
\text { unknown origin of disease (2) }\end{array}$ \\
\hline
\end{tabular}

$A D P K D$ autosomal dominant polycystic kidney disease, CSC central serous chorioretinopathy, FA fluorescein angiography, $O C T$ optical coherence tomography, $S R F$ subretinal fluid

* Outer photoreceptor/retinal pigment epithelium changes on OCT, reminiscent of changes in chronic CSC, with corresponding hyperfluorescent changes on FA an increase in SFCT, comparison of the measurements in our patients with matched healthy controls to whom low-dose steroids had not been prescribed chronically could have attached significance to our data $[25,26]$.

Even though we expected to find abnormal ocular findings in patients with chronic kidney disease, given the correlation between nephrological and ophthalmological diseases [27-29], the prevalence of abnormalities was higher than we had expected beforehand. This may have been caused by the use of other (immunosuppressive) medication, due to hypertension, due to pre-existing renal diseases, or even due to diabetes mellitus.

In conclusion, retinal abnormalities are present in the majority of renal transplant patient who use low-dose steroids chronically. Therefore, an ophthalmological examination including evaluation of the retina is indicated in these patients. However, seeing that the ophthalmological abnormalities were relatively mild and had a low likelihood for causing symptoms and complications, the dosage of prescribed steroids does not have to be lowered or discontinued in this patient group.

Acknowledgments This research was supported by the following foundations: MaculaFonds, Retina Netherlands, BlindenPenning, and Landelijke Stichting voor Blinden en Slechtzienden, which contributed through UitZicht, as well as Rotterdamse Stichting Blindenbelangen, Stichting Blindenhulp, ZonMw VENI Grant, and Gisela Thier Fellowship of Leiden University (CJFB). The funding organizations had no role in the design or conduct of this research. They provided unrestricted grants.

Compliance with ethical standards All procedures performed were in accordance with the ethical standards of the institutional and/or national research committee and with the 1964 Helsinki Declaration and its later amendments or comparable ethical standards. 
Informed consent Informed consent was obtained from all individual participants included in the study.

Conflict of interest All authors certify that they have no affiliations with or involvement in any organization or entity with any financial interest (such as honoraria; educational grants; participation in speakers' bureaus; membership, employment, consultancies, stock ownership, or other equity interest; and expert testimony or patent-licensing arrangements), or non-financial interest (such as personal or professional relationships, affiliations, knowledge, or beliefs) in the subject matter or materials discussed in this manuscript.

Open Access This article is distributed under the terms of the Creative Commons Attribution 4.0 International License (http:// creativecommons.org/licenses/by/4.0/), which permits use, duplication, adaptation, distribution and reproduction in any medium or format, as long as you give appropriate credit to the original author(s) and the source, provide a link to the Creative Commons license, and indicate if changes were made.

\section{References}

1. Jahadi-hosseini HR, Rahmani B, Karbassi A, Mehrian M, Medghalchi AR (2003) Ocular complications in renal allograft recipients. Transplant Proc 35:309-310

2. Aydin P, Oto S, Kadayifcilar S, Dursun D (1996) Ophthalmological problems in renal transplant patients. Transplant Proc 28:2312-2313

3. Su CC, Chen JW, Chou NK, Chen YS, Huang SC, Chi NH, Wang SS (2014) Ocular manifestations of patients receiving heart transplants: a single-center experience of 311 consecutive cases. Transplant Proc 46:937-940. https://doi.org/10.1016/j. transproceed.2013.11.149

4. Moon SJ, Mieler WF (2003) Retinal complications of bone marrow and solid organ transplantation. Curr Opin Ophthalmol 14:433-442

5. Jayamanne DG, Porter R (1998) Ocular morbidity following renal transplantation. Nephrol Dial Transplant 13:2070-2073

6. Klaver CC, Hoyng CB, de Jong PT (1995) Pigmentary irregularities and optic disc edema after heart transplantation. Arch Ophthalmol 113:1281-1285

7. Boon CJ, Klevering BJ, Leroy BP, Hoyng CB, Keunen JE, den Hollander AI (2009) The spectrum of ocular phenotypes caused by mutations in the Best1 gene. Prog Retin Eye Res 28:187-205. https://doi.org/10.1016/j.preteyeres.2009.04.002

8. Fawzi AA, Holland GN, Kreiger AE, Heckenlively JR, Arroyo JG, Cunningham ET Jr (2006) Central serous chorioretinopathy after solid organ transplantation. Ophthalmology 113:805-813. https:// doi.org/10.1016/j.ophtha.2006.01.031

9. Polak BC, Baarsma GS, Snyers B (1995) Diffuse retinal pigment epitheliopathy complicating systemic corticosteroid treatment. Br J Ophthalmol 79:922-925

10. Lee CS, Kang EC, Lee KS, Byeon SH, Koh HJ, Lee SC (2011) Central serous chorioretinopathy after renal transplantation. Retina 31:1896-1903. https://doi.org/10.1097/IAE.0b013e31820a69ee

11. Kian-Ersi F, Taheri S, Akhlaghi MR (2008) Ocular disorders in renal transplant patients. Saudi J Kidney Dis Transpl 19:751-755

12. Carvalho-Recchia CA, Yannuzzi LA, Negrao S, Spaide RF, Freund KB, Rodriguez-Coleman H, Lenharo M, Iida T (2002) Corticosteroids and central serous chorioretinopathy. Ophthalmology 109:1834-1837
13. Bouzas EA, Karadimas P, Pournaras CJ (2002) Central serous chorioretinopathy and glucocorticoids. Surv Ophthalmol 47:431-448

14. Yannuzzi LA (1987) Type-a behavior and central serous chorioretinopathy. Retina 7:111-131

15. Bousquet E, Beydoun T, Zhao M, Hassan L, Offret O, Behar-Cohen F (2013) Mineralocorticoid receptor antagonism in the treatment of chronic central serous chorioretinopathy: a pilot study. Retina 33: 2096-2102. https://doi.org/10.1097/IAE.0b013e318297a07a

16. Dalvin LA, Fervenza FC, Sethi S, Pulido JS (2016) Manifestations of complement-mediated and immune complex-mediated membranoproliferative glomerulonephritis: a comparative consecutive series. Ophthalmology 123:1588-1594. https://doi.org/10 1016/j.ophtha.2016.02.018

17. Gehrs KM, Pollock SC, Zilkha G (1995) Clinical features and pathogenesis of Alport retinopathy. Retina 15:305-311

18. Haimovici R, Koh S, Gagnon DR, Lehrfeld T, Wellik S (2004) Risk factors for central serous chorioretinopathy: a case-control study. Ophthalmology 111:244-249. https://doi.org/10.1016/j.ophtha. 2003.09.024

19. Jonas JB, Kamppeter BA (2005) Intravitreal triamcinolone acetonide and central serous chorioretinopathy. Br J Ophthalmol 89:386-387. https://doi.org/10.1136/bjo.2004.054247

20. Chung YR, Kim JW, Kim SW, Lee K (2016) Choroidal thickness in patients with central serous chorioretinopathy: assessment of Haller and Sattler layers. Retina 36:1652-1657. https://doi.org/10.1097/ iae.0000000000000998

21. Imamura Y, Fujiwara T, Margolis R, Spaide RF (2009) Enhanced depth imaging optical coherence tomography of the choroid in central serous chorioretinopathy. Retina 29:1469-1473. https://doi.org/ 10.1097/IAE.0b013e3181be0a83

22. Yang L, Jonas JB, Wei W (2013) Optical coherence tomographyassisted enhanced depth imaging of central serous chorioretinopathy. Invest Ophthalmol Vis Sci 54:4659-4665. https://doi.org/10.1167/iovs.12-10991

23. Prunte C, Flammer J (1996) Choroidal capillary and venous congestion in central serous chorioretinopathy. Am J Ophthalmol 121: 26-34

24. Gill GN (1990) The adrenal gland. In: West JB (Ed): Best and Taylor's physiological basis of medical practice, 12th edn. Williams and Wilkins, Baltimore

25. Wei WB, Xu L, Jonas JB, Shao L, Du KF, Wang S, Chen CX, Xu J, Wang YX, Zhou JQ, You QS (2013) Subfoveal choroidal thickness: the Beijing Eye Study. Ophthalmology 120:175-180. https://doi. org/10.1016/j.ophtha.2012.07.048

26. Ramrattan RS, van der Schaft TL, Mooy CM, de Bruijn WC, Mulder PG, de Jong PT (1994) Morphometric analysis of Bruch's membrane, the choriocapillaris, and the choroid in aging. Invest Ophthalmol Vis Sci 35:2857-2864

27. Grunwald JE, Alexander J, Maguire M, Whittock R, Parker C, McWilliams K, Lo JC, Townsend R, Gadegbeku CA, Lash JP, Fink JC, Rahman M, Feldman H, Kusek J, Ojo A (2010) Prevalence of ocular fundus pathology in patients with chronic kidney disease. Clin J Am Soc Nephrol 5:867-873. https://doi. org/10.2215/cjn.08271109

28. Wong TY, Coresh J, Klein R, Muntner P, Couper DJ, Sharrett AR, Klein BE, Heiss G, Hubbard LD, Duncan BB (2004) Retinal microvascular abnormalities and renal dysfunction: the atherosclerosis risk in communities study. J Am Soc Nephrol 15:2469-2476. https://doi.org/10.1097/01.asn.0000136133.28194.e4

29. Sabanayagam C, Shankar A, Koh D, Chia KS, Saw SM, Lim SC, Tai ES, Wong TY (2009) Retinal microvascular caliber and chronic kidney disease in an Asian population. Am J Epidemiol 169:625632. https://doi.org/10.1093/aje/kwn367 